\title{
Adjuvant Chemotherapy for Gastric Cancer Patients with Mismatch Repair Deficiency or Microsatellite Instability: Systematic Review and Meta-Analysis
}

\section{Runcong Nie}

Sun Yat-sen University Cancer Center

\section{Shuqiang Yuan}

Sun Yat-sen University Cancer Center

Jin Won Kim

Seoul National University Bundang Hospital, Seoul National University College of Medicine

\section{Yun Wang}

Sun Yat-sen University Cancer Center

\section{Guoming Chen}

Sun Yat-sen University Cancer Center

Jie Zhou

Sun Yat-sen University Cancer Center

\section{Yingbo Chen}

Sun Yat-sen University Cancer Center

\section{Shi Chen}

Sun Yat-sen University

\section{Zhiwei Zhou}

Sun Yat-sen University Cancer Center

\section{Muyan Cai}

Sun Yat-sen University Cancer Center

Yuanfang Li ( $\nabla$ liyuanf@sysucc.org.cn )

Sun Yat-sen University Cancer Center

\section{Research Article}

Keywords: Mismatch repair deficient (dMMR), microsatellite instability-high (MSI-H)

Posted Date: April 29th, 2021

DOl: https://doi.org/10.21203/rs.3.rs-441580/v1 
License: (c) (i) This work is licensed under a Creative Commons Attribution 4.0 International License. Read Full License

Version of Record: A version of this preprint was published at Annals of Surgical Oncology on November 18th, 2021. See the published version at https://doi.org/10.1245/s10434-021-11050-6. 
Adjuvant chemotherapy for gastric cancer patients with mismatch repair deficiency or microsatellite instability: systematic review and meta-analysis

\section{Running title: Adjuvant chemotherapy for dMMR/MSI-H gastric cancer}

Runcong Nie, MD, PhD ${ }^{1 \#}$; Shuqiang Yuan, MD, PhD ${ }^{1 \#}$; Jin Won Kim, MD, PhD ${ }^{2 \#}$; Yun Wang, MD, PhD ${ }^{3 \#}$; Guoming Chen, $\mathrm{MD}^{1 \#}$; Jie Zhou ${ }^{4 \#}$, Yingbo Chen, $\mathrm{MD}^{1}$; Shi Chen, MD, PhD ${ }^{5}$; Zhiwei Zhou, MD, $\mathrm{PhD}^{1 *}$; Muyan Cai, MD, $\mathrm{PhD}^{6 *}$; Yuanfang Li, $\mathrm{MD}, \mathrm{PhD}^{1^{*}}$

1 Department of Gastric Surgery, Sun Yat-sen University Cancer Center; State Key Laboratory of Oncology in South China; Collaborative Innovation Center for Cancer Medicine, Guangzhou, P. R. China

2 Division of Hematology and Medical Oncology, Department of Internal Medicine; Seoul National University Bundang Hospital, Seoul National University College of Medicine, Seongnam, Korea

3 Department of Hematologic Oncology, Sun Yat-sen University Cancer Center; State Key Laboratory of Oncology in South China; Collaborative Innovation Center for Cancer Medicine, Guangzhou, P. R. China 4 State Key Laboratory of Oncology in South China, Collaborative Innovation Center for Cancer Medicine, Sun Yat-sen University Cancer Center, Guangzhou, P. R. China

5 Department of Gastrointestinal Surgery, the Sixth Affiliated Hospital, Sun Yat-sen University, Guangzhou, P. R. China

6 Department of Pathology, Sun Yat-sen University Cancer Center; State Key Laboratory of Oncology in South China; Collaborative Innovation Center for Cancer Medicine, Guangzhou, P. R. China

${ }^{\#}$ These authors contributed equally to this study; ${ }^{*}$ These authors are co-senior authors.

The authors declare no potential conflicts of interest.

\section{*Corresponding Authors:}

Yuan-Fang Li, Department of Gastric Surgery, Sun Yat-sen University Cancer Center, No. 651 Dongfeng Eastern Road, Guangzhou, Guangdong, 510060, P. R. China (liyuanf@sysucc.org.cn); Mu-Yan Cai, Department of Pathology, Sun Yat-sen University Cancer Center, No. 651 Dongfeng Eastern Road, 
Guangzhou, Guangdong, 510060, P. R. China (caimy@sysucc.org.cn); Zhi-Wei Zhou, Department of Gastric Surgery, Sun Yat-sen University Cancer Center, No. 651 Dongfeng Eastern Road, Guangzhou, Guangdong, 510060, P. R. China (zhouzhw@sysucc.org.cn);

Manuscript word count: 2820 


\begin{abstract}
Background: Mismatch repair deficient (dMMR) or microsatellite instability-high (MSI-H) serves as a predictor poor response to adjuvant chemotherapy in stage II colon cancer patients. Our objective was to investigate the efficacy of adjuvant chemotherapy in dMMR/MSI-H gastric cancer (GC).

Methods: We searched literatures through December, 2020 to identify clinical studies that reported survival comparing adjuvant chemotherapy with surgery alone in dMMR/MSI-H GCs. Two approaches were used to pool the hazard ratio (HR) of survival: (1) If Kaplan-Meier curves and number at risk were provided, individual patient data were extracted. Cox models were used to calculate the HR with $95 \%$ confidence interval (CI); (2) for study-level data, pooled HR was estimated using fixed/random-effects models.

Results: Six clinical studies were identified. For dMMR/MSI-H versus mismatch repair proficient (pMMR)/microsatellite stable (MSS)/microsatellite instability-low (MSI-L), the estimated 5-years DFS were $74.2 \%$ versus $51.5 \%$ (HR, 0.44; 95\% CI, 0.32-0.62; $P<0.001$ ); the estimated 5-years OS were $60.8 \%$ versus $50.1 \%$ (HR, 0.72; 95\% CI, 0.60-0.88; $P=0.001)$. At study-level data, the pooled HRs were 0.42 for DFS (95\% CI, $0.31-0.57 ; P<0.001)$ and 0.66 for OS $(95 \% \mathrm{CI}, 0.32-1.38 ; P=0.268)$. For adjuvant chemotherapy versus observation in dMMR/MSI-H, the estimated 5-years DFS were $76.1 \%$ versus $73.3 \%$ (HR, 0.72; 95\% CI, 0.45 $1.15 ; P=0.171)$; the estimated 5 -years OS were $74.9 \%$ versus $60.2 \%(\mathrm{HR}, 0.60 ; 95 \% \mathrm{CI}, 0.44-0.83 ; P=$ 0.001). Significant survival differences were also observed at study-level.
\end{abstract}

Conclusions: This study further suggested adjuvant chemotherapy could be beneficial even in dMMR/MSIH GC patients. 


\section{Introduction}

Over 1 billion new gastric cancer (GC) cases and 783000 deaths are estimated to occur globally in 2018, ranking GC as the fifth most frequently diagnosed cancer and the third leading cancer-related death worldwide $[1,2]$. In Asia, adjuvant chemotherapy with S1 [3, 4] or capecitabine plus oxaliplatin $[5,6]$ is the current standard of care to prevent recurrence and improve survival after curative D2 gastrectomy for stage II-III GC patients [7]. However, approximately $40 \%$ of patients with primary resected GC receiving adjuvant chemotherapy still suffer from relapse and/or metastases $[4,6,8]$. Thus, predictive tools able to identify patients who would possibly benefit from adjuvant chemotherapy are urgently needed. Cheong et al. reported that four classifier genes $(G Z M B, W A R S, S F R P 4$, and $C D X 1)$ could serve as a prognostic and predictive tool to predict the survival and adjuvant chemotherapy response after surgery in stage II-III GCs [9]. Recently, Wang et al. established a novel score system based on 6 immune checkpoints to assist the adjuvant chemotherapy selection in GCs [10].

Tumors that are deficient in DNA mismatch repair (dMMR) loss the ability of cells to recognize and repair spontaneous DNA mutations [11]. dMMR tumors have distinct characteristics such as high tumor mutation burden and hypermutator phenotype, termed microsatellite instability-high (MSI-H) [12]. Prior studies have indicated that dMMR/MSI-H correlates with improved survival in resectable colon cancer and serves as a predictor of poor response to adjuvant chemotherapy in stage II colon cancer patients [13, 14]. The frequency of $\mathrm{dMMR} / \mathrm{MSI}-\mathrm{H}$ has been found to be relatively high in $\mathrm{GC}, \sim 9 \%-22 \%$ of all GC cases $[12,15]$, therefore, raising the debatable issue: whether dMMR/MSI-H could serve as a predictor to distinguish whom cannot benefit from adjuvant chemotherapy.

Both the post hoc of the CLASSIC study [16] and individual patient data (IPD) from 4 randomized trials (MAGIC, CLASSIC, ARTIST and ITACA-S) [17] showed that patients with MSI-H did not benefit from adjuvant chemotherapy. However, considering the small sample of patients $(n=61)$ with MSI-H in the MAGIC and CLASSIC trials which could have largely reduced statistical power, and examination of the resected rather than biopsy specimens in the chemotherapy arm of MAGIC study indicated that pathological major or complete responders were not included, these suggest a certain level of result bias. Subsequently, a recent large cohort study comprising of 162 MSI-H patients with GC revealed that adjuvant chemotherapy, compared with surgery alone, was associated with longer disease-free survival (DFS) and overall survival (OS) [18]. Thus, the significance of adjuvant chemotherapy in dMMR/MSI-H GC is yet to be fully elucidated.

In this study, we performed a systematic review and meta-analysis, at individual- and study- levels, to investigate whether resectable GC patients with dMMR/MSI-H could benefit from adjuvant chemotherapy. The prognostic value of dMMR/MSI-H versus mismatch repair proficient (pMMR), microsatellite stable 
(MSS), and microsatellite instability-low (MSI-L) in gastric cancer was also explored.

\section{Methods}

\section{Search strategy and selection criteria}

Two investigators (R.C.N. and S.Q.Y.) searched the PubMed, Web of Science and Embase databases till December 18th, 2020, for clinical studies that reported on the survival outcomes of adjuvant chemotherapy versus surgery alone in $\mathrm{dMMR}$ or MSI-H GC after radical resection. The key words used were gastric cancer, adjuvant chemotherapy and $d M M R$ or $M S I-H$. The detailed search terms are displayed in online supplemental Box 1. This study was conducted in compliance with the PRISMA statement guidelines for systematic reviews [19].

We excluded studies which were reviews, conference abstracts, case reports, and abstracts. If duplicated studies were reported, we only included their most recent reports which had complete data. Disagreements were resolved by consulting a senior investigator (Y.F.L.).

\section{Data extraction and quality assessment}

R.C.N. and G.M.C. extracted the following information from included studies if available: study type, study period, number of dMMR/MSI-H gastric cancer cases, number of adjuvant chemotherapy versus surgery alone in dMMR/MSI-H patients. Subsequently, survival rates and hazard ratio (HR) with $95 \%$ confidence interval (CI) of adjuvant chemotherapy versus surgery alone of the dMMR/MSI-H patients, and/or dMMR/MSI-H versus pMMR/MSS/MSI-L were extracted. The detailed information of MSI status, chemotherapy, and survival in the study of Wang et al. [10] is shown in the Supplementary Data.

The qualities of randomised controlled trials (RCTs) and retrospective studies were assessed using the Cochrane risk of bias tool [20] and Newcastle-Ottawa scale [21], respectively. A score of 0-9 was allocated to each retrospective study. RCTs and retrospective studies with a score higher than 6 points were considered as high quality.

\section{Killing activity of chemotherapy drugs in gastric cancer cell lines}

PRISM is a molecular barcoding method to test a total of 4518 drugs against 578 human cancer cell lines in pools [22]. The primary PRISM Repurposing dataset that screened each drug in triplicate at a single dose $(2.5 \mu \mathrm{M})$ was included to analyze the killing ability of chemotherapy agents in MSI-H and MSS GC cell lines (https://depmap.org/repurposing). 
A total of $20 \mathrm{GC}$ cell lines, comprising of $3 \mathrm{MSI}-\mathrm{H}$ and $17 \mathrm{MSS}$, were included (online supplemental table S1). The chemotherapy agents that had been recommended for adjuvant therapy in GCs after radical surgery were included; thus, a total of 12 agents were eligible (online supplemental table S2). The killing activity was quantified as $\log 2$ fold change values relative to DMSO.

\section{Statistical analysis}

The primary endpoints were DFS and OS differences of the adjuvant chemotherapy after surgery versus surgery alone in $\mathrm{dMMR} / \mathrm{MSI}-\mathrm{H}$ patients. We also assessed the survival differences of $\mathrm{dMMR} / \mathrm{MSI}-\mathrm{H}$ versus pMMR/MSS/MSI-L patients. In this study, we included relapse-free survival in the definition of DFS. We used 2 different approaches to pool the HR of survival: (1) if the Kaplan-Meier curves and number at risk information were provided, IPD were extracted using graph digitizer software (Engauge Digitizer 12.1) and “reconstructKM" package in R (https://github.com/ryanrsun/reconstructKM); then, the survival curves were estimated using the Kaplan-Meier method, and Cox proportional hazard regression models were used to estimate the HR; (2) for study-level data, the HR was estimated by pooling the HR of each eligible study. The odds ratio (OR) was used to explore the associations between dMMR/MSI-H and clinicopathological characteristics. We used the Cochrane $Q$ test and the $I^{2}$ statistic to assess the level of heterogeneity among studies, with $P<0.10$ and $I^{2}$ greater than $50 \%$ considered significant heterogeneity. The random-effects models were chosen if heterogeneity was observed, otherwise, fixed-effects models were used.

A 2-sided $P$ value $<0.05$ was considered statistically significant. All analyses were performed using statistical software R version 3.6.1 (R Foundation for Statistical Computing; http://www.r-project.org).

\section{Results}

\section{Study characteristics}

Initially, a total of 338 records were retrieved after literature search of databases. Subsequently, 127 records were excluded because of duplication, 196 records were excluded after screening titles and abstracts, and 9 studies were excluded after testing the full-text articles. Finally, a total of 6 studies [10, 16, 18, 23-25] were identified for quantitative analysis (figure 1).

Overall, the meta-analysis comprised 5 retrospective studies and 1 RCT. The percentage of dMMR/MSI$\mathrm{H}$ ranged from $6.8 \%$ to $26.3 \%$. Kim et al. [18] reported two cohorts, one of which included to analyze the survival of MSI-H compared with MSS/MSI-L. The other cohort was included to analyze the efficacy of adjuvant chemotherapy in MSI-H patients. Three of the included studies were identified as high quality (online 
supplemental table S3).

\section{Association between dMMR/MSI-H and clinicopathological characteristics}

The correlations between $\mathrm{dMMR} / \mathrm{MSI}-\mathrm{H}$ and clinicopathological characteristics were investigated (online supplemental table S4). Pooled analyses found no significant correlation between dMMR/MSI-H and gender (OR, 0.98; 95\% CI, 0.58-1.65; $P=0.933)$, and incidence of dMMR/MSI-H was greater in patients with intestinal Laurén classification (OR, 2.56; 95\% CI, 1.83-3.58; $P<0.001)$, lower tumor location (OR, 2.53; 95\% CI, 1.76-3.63; $P<0.001)$, and pathological stage I-II (OR, 1.62; 95\% CI, 1.15-2.29; $P=0.006)$.

\section{Survival of dMMR/MSI-H}

The survival curves for dMMR/MSI-H and pMMR/MSS/MSI-L are shown in figure 2. Three of the included studies $[16,18,25]$ comprising of 1756 patients reported IPD of DFS. The estimated 3-, 5-, and 10-years DFS of patients with dMMR/MSI-H were 78.4\% (95\% CI, 71.9\%-85.5\%), 74.2\% (95\% CI, 67.1\%-81.9\%) and $72.6 \%$ (95\% CI, 65.2\%-80.9\%), while that of patients with pMMR/MSS/MSI-L were $58.0 \%$ (95\% CI, 71.9\%$85.5 \%), 51.5 \%$ (95\% CI, 48.9\%-54.0\%) and 45.9\% (95\% CI, 42.9\%-49.1\%). The estimated HR for DFS was 0.44 (95\% CI, 0.32-0.62; $P<0.001$ ) (figure 2A). Similarly, the 3-, 5-, and 10-year OS of patients with $\mathrm{dMMR} / \mathrm{MSI}-\mathrm{H}$ was superior than that of patients with pMMR/MSS/MSI-L (68.2\% [95\% CI, 63.0\%-73.9\%] vs $58.0 \%$ [95\% CI, 55.6\%-60.6\%], 60.8\% [95\% CI, 55.3\%-66.9\%] vs 50.1\% [95\% CI, 47.6\%-52.7\%], and 56.6\% [95\% CI, 50.7\%-63.2\%] vs 41.2\% [95\% CI, 38.2\%-44.4\%], respectively). The estimated HR for OS was $0.72(95 \% \mathrm{CI}, 0.60-0.88 ; P=0.001)$ (figure 2B).

The pooled HRs of dMMR/MSI-H versus pMMR/MSS/MSI-L at study-level data are shown in figure 3. No significant heterogeneity was observed in pooling the HR for DFS; thus, the pooled results were based on the fixed-effects model. Corresponding forest plot showed that patients with dMMR/MSI-H had a significantly prolonged DFS (HR, 0.42; 95\% CI, 0.31-0.57; $P<0.001$; figure 3A), compared with those with pMMR/MSS/MSI-L. Random-effects model was performed to estimate the pooled HR for OS because the Cochrane $Q$ test and the $I^{2}$ statistic showed significant heterogeneity. The estimated HR for OS was 0.66 (95\% CI, 0.32-1.38; $P=0.268$ ) (figure 3B).

\section{Survival in dMMR/MSI-H patients treated with adjuvant chemotherapy}

Next, the efficacy of adjuvant chemotherapy in dMMR/MSI-H was investigated and the survival curves are presented in figure 4. Four studies $[16,18,24,25]$ were included to collect the IPD of DFS. Although small 
sample size in dMMR/MSI-H reduced the power to reach statistic difference (HR, 0.72; 95\% CI, 0.45-1.15; $P=0.171$; figure 4A), the DFS of patients receiving adjuvant chemotherapy was longer than those receiving surgery alone, with 3-, 5-, and 10-years DFS of (82.4\% [95\% CI, 76.5\%-88.8\%] vs 75.5\% [95\% CI, 68.0\%83.9\%], 76.1\% [95\% CI, 69.1\%-83.7\%] vs 73.3\% [95\% CI, 65.5\%-82.0\%], and $73.2 \%$ [95\% CI, 65.6\%-81.7\%] vs $67.3 \%$ [95\% CI, 58.3\%-78.3\%], respectively).

Next, we collected a total of 437 IPD of OS from 4 studies $[10,18,24,25]$. The larger sample size improved the statistical power, and statistically prolonged OS was observed (HR, 0.60; 95\% CI, 0.44-0.83; $P$ $=0.001$; figure $4 \mathrm{~B})$, with 3-, 5-, and 10-years OS of 81.9\% (95\% CI, 77.0\%-87.1\%), 74.9\% (95\% CI, 69.4\%$80.8 \%)$ and $65.3 \%$ (95\% CI, 57.9\%-73.7\%) in patients receiving adjuvant chemotherapy versus $65.9 \%$ (95\% CI, 59.7\%-72.8\%), 60.2\% (95\% CI, 53.7\%-67.4\%) and 50.6\% (95\% CI, 42.2\%-60.6\%) in patients receiving surgery alone.

Finally, the efficacy of adjuvant chemotherapy in dMMR/MSI-H patients at study-level data was explored (figure 5). The pooled results were assessed by the fixed-effects model because the Cochrane $Q$ test and the $I^{2}$ statistic showed homogeneity. The forest plot showed that the pooled HRs for adjuvant chemotherapy after surgery versus surgery alone revealed that dMMR/MSI-H patients could benefit from adjuvant chemotherapy, with estimated HRs of 0.56 (95\% CI, 0.36-0.87; $P=0.010$; figure 5A) for DFS and 0.59 (95\% CI, 0.43-0.82; $P=0.002$; figure 5B) for OS.

\section{Killing activity of chemotherapy drugs in cell lines}

Analyzing the primary PRISM Repurposing dataset [22], we found that the killing ability of fluorouracil drugs was weak and similar between MSI and MSS GC cell lines (online supplemental figure S1A-E). The killing ability of cisplatin in MSI cell lines was negligible (figure S1F). However, the killing ability of oxaliplatin (figure S1G-H), paclitaxel (figure S1I), docetaxel (figure S1J-K) and irinotecan (figure S1L) in MSI cell lines were robust, especially in one of the docetaxel drugs (BRD-K30577245-341-01-9, figure S1J).

\section{Discussion}

To the best of our knowledge, this study is the first systematic review and meta-analysis to investigate the efficacy of adjuvant chemotherapy in GC with dMMR/MSI-H. Using the published data from 6 studies, our pooled analysis suggested that patients with dMMR/MSI-H had superior DFS and OS, compared to those with

pMMR/MSS/MSI-L. Of note, DFS and OS were prolonged in dMMR/MSI-H patients treated with adjuvant chemotherapy, compared to those treated with surgery alone. The killing activities of 12 chemotherapy drugs 
in MSI-H GC cell lines were weak to strong. These findings indicated that GC patients with dMMR/MSI-H could still benefit from adjuvant chemotherapy after radical resection.

MMR-deficiency or MSI-H is one of the four major subtypes in gastric cancer [26]. Tumors with dMMR/MSI-H have distinct clinical and pathological features. A previous meta-analysis [27] showed that $\mathrm{dMMR} / \mathrm{MSI}-\mathrm{H}$ tumors occurred more frequently in female and elderly patients, tumors located at the lower $1 / 3$ of the stomach with intestinal Laurén classification and early TNM stage. Our study indicated the coincident results. Pooling the individual- and study-level data, our study further confirmed the positive prognostic effect of dMMR/MSI-H status with respect to DFS and OS, which was consistent with previous studies $[17,27,28]$. A higher percentage of Laurén intestinal subtype and early stage in dMMR/MSI-H could indicate that dMMR/MSI-H could serve as a confounder rather than prognostic factor. However, IPD showed that the pooled HRs of dMMR/MSI-H for DFS (0.42) and OS (0.72) were quite low. Kim et al. also reported that MSI-H status was a prognostic marker of DFS (HR, $0.40 ; P=0.059)$ and OS $(\mathrm{HR}, 0.40 ; P=0.063)$ in the multivariate analysis. Therefore, we believed that $\mathrm{dMMR} / \mathrm{MSI}-\mathrm{H}$ is a prognostic marker, not a confounder.

The invalid effect of adjuvant chemotherapy in dMMR/MSI-H colon cancer raised the hypothesis that resected GCs with dMMR/MSI-H had poor response from adjuvant chemotherapy. Recently, Pietrantonio et al. [17] reported the IPD from 4 randomized trials. Their results showed that MSI-H GCs could not benefit from adjuvant chemotherapy, based on which, the 2020 clinical guideline of the Chinese Society of Clinical Oncology (CSCO) for the diagnosis and treatment of gastric cancer has recommended that MSI-H patients after radical surgery are not encouraged to receive adjuvant chemotherapy, but to observe or participate in clinical trials (Grade II recommendation; Evidence 1B). However, the small sample size in this study of Pietrantonio et al. [17] significantly reduced the power, which limited the guidance of adjuvant chemotherapy based on the status of MMR or MSI. Our study is the largest cohort of individual- and study-level data of dMMR/MSI-H patients. Our findings revealed that adjuvant chemotherapy could improve the DFS and OS of dMMR/MSI-H patients. In addition, we found that the anti-tumor activities of oxaliplatin, paclitaxel, docetaxel and irinotecan in MSI gastric cancer cell lines were significant. Therefore, we should not justify a change in the clinical practice so far. Based on our findings, we suggest that adjuvant chemotherapy, rather than observation, should be recommended to $\mathrm{dMMR} / \mathrm{MSI}-\mathrm{H}$ patients after radical surgery.

Moving forward, one new dilemma that could be raised is whether adjuvant chemotherapy is the optimal therapeutic strategy for GC patients with dMMR/MSI-H. It is recognized that tumors with dMMR/MSI-H were associated with many somatic mutations that encode "non-self" immunogenic neoantigens. These tumors were accompanied by infiltrative immune cells and high tumor mutation burden, and thus were susceptible to 
immunotherapy. Programmed death 1 (PD-1) blockade has demonstrated clinical benefit for patients with dMMR/MSI-H regardless of tumor types [29-31]. Based on the results of the KEYNOTE-059 that showed effective objective response rate (ORR: 57.0\%, 4 of 7 patients) in GCs with dMMR/MSI-H [32], the US FDA approved pembrolizumab as second-line treatment for these patients. Two phase 2 clinical trials that included GC patients who were treated with pembrolizumab as $\geq 2$ lines of treatment also demonstrated effective clinical response in patients with dMMR/MSI-H, with ORR of 85.7\% (6 of 7) [33] and 45.8\% (11 of 24) [15], respectively. Based on such premises, adjuvant treatment using PD-1 inhibitors could be theoretically superior to chemotherapy for dMMR/MSI-H GC patients after radical surgery. Therefore, prospective clinical trials to investigate the efficacy of adjuvant PD-1 blockade compared with chemotherapy in dMMR/MSI-H GCs are urgently needed.

A major limitation of this study was that the eligible studies were retrospective studies or post hoc analysis of clinical trial. The heterogeneity among studies could have affected our results, to a certain extent. Thus, random-effects models were performed when heterogeneity was observed, and the large sample size used improved the statistical power and reliability of our analysis. Despite that, head-to-head prospective randomized control trial is still needed to clarify the efficacy of adjuvant chemotherapy in patients with $\mathrm{dMMR} / \mathrm{MSI}-\mathrm{H}$. In the era of immunotherapy, we believe that prospective trials should compare the efficacy of adjuvant PD-1 blockade with chemotherapy in patients with dMMR/MSI-H. Finally, only the PRISM Repurposing dataset was used to analyze the anti-tumor activity of chemotherapy in MSI-H cell lines, and in vitro experiments to further clarify the potential underlying mechanisms are still needed.

In conclusions, our meta-analysis suggests that GC patients with dMMR/MSI-H had better survival than those with pMMR/MSS/MSI-L. Adjuvant chemotherapy could prolong the survival of dMMR/MSI-H patients compared to observation only. Thus, chemotherapy should not be omitted for dMMR/MSI-H patients after surgery. In the era of immunotherapy, adjuvant PD-1 blockade should be investigated prospectively in dMMR/MSI-H patients, but chemotherapy should still be set as the control arm.

\section{Figure legend}




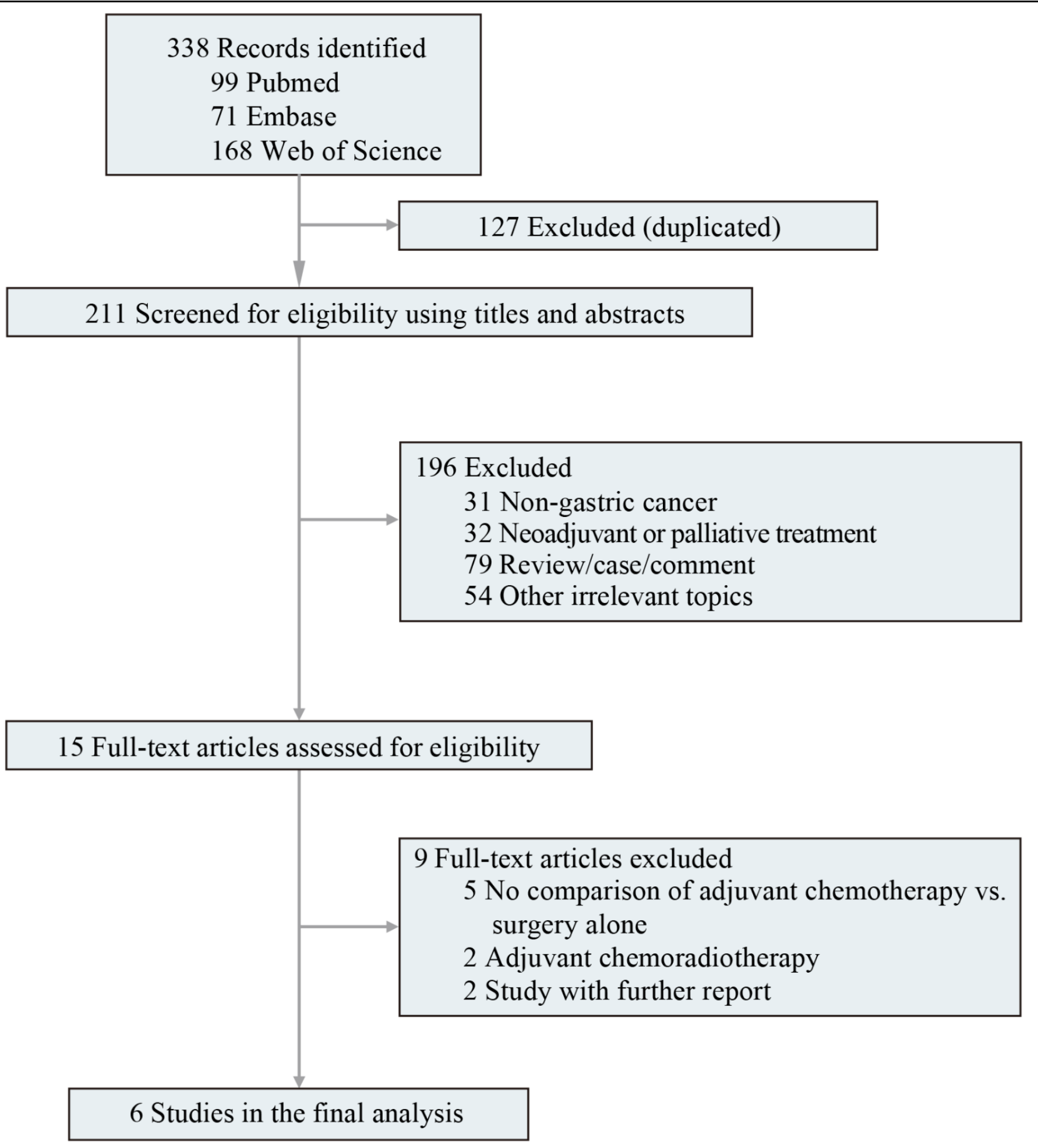

Figure 1. Study flow diagram. Six studies were identified for quantitative analysis. 
$A$

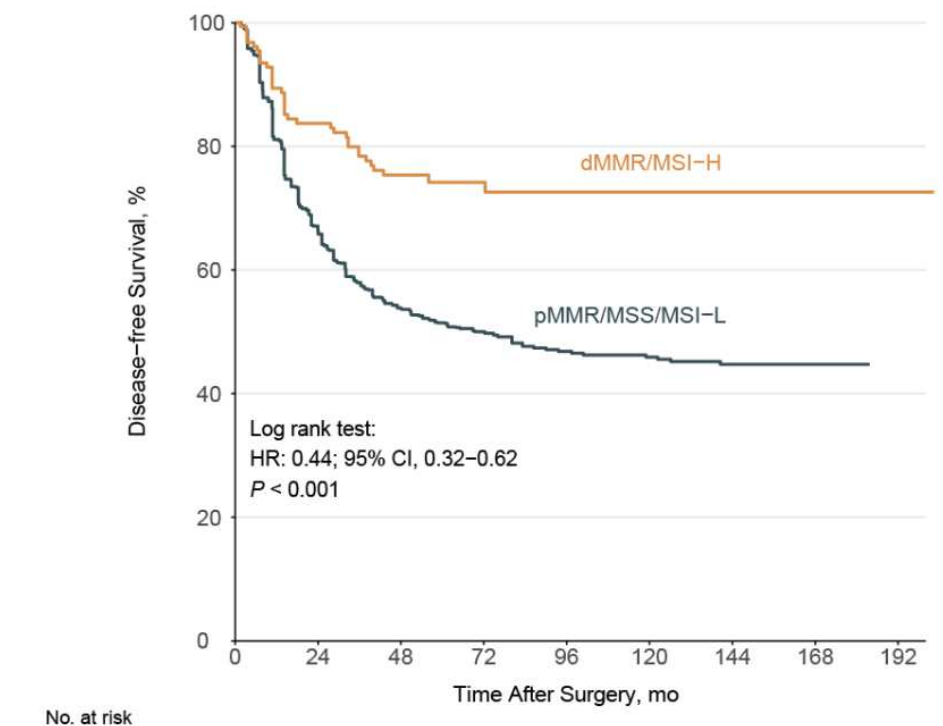

$\begin{array}{llllllllll}\text { pMMR/MSS/MSI-L } 1597 & 964 & 726 & 378 & 163 & 134 & 88 & 79 & 0\end{array}$

$\begin{array}{llllllllll}\text { dMMR/MSI-H } & 159 & 111 & 97 & 47 & 23 & 12 & 10 & 4 & 1\end{array}$
B

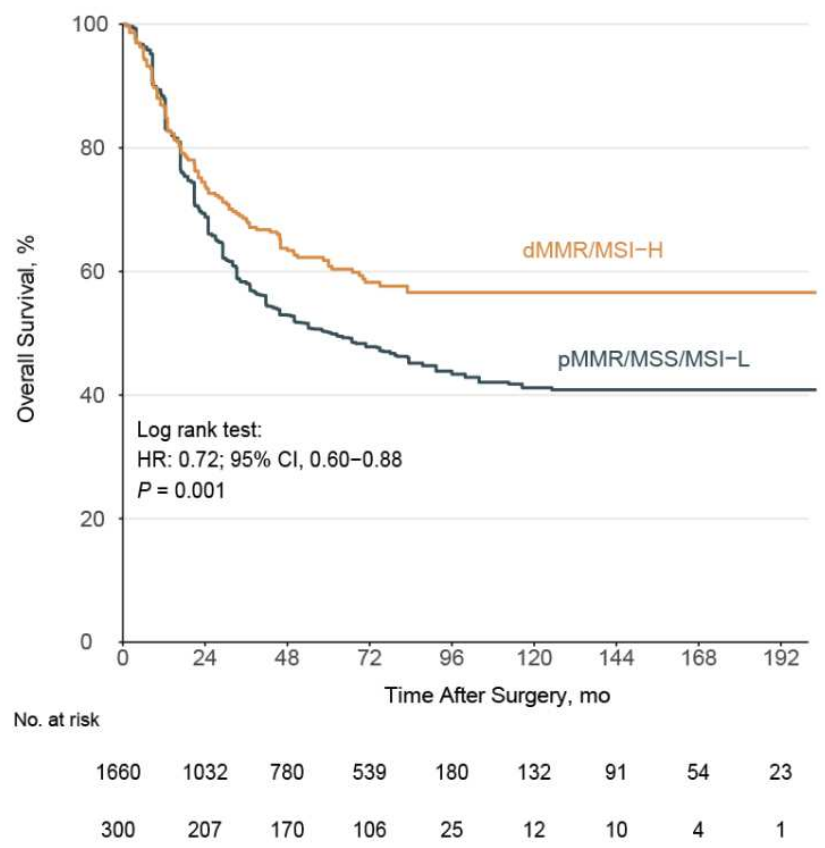

Figure 2. Individual patient data of disease-free survival (A) and overall survival (B) for gastric cancer patients with dMMR/MSI-H versus pMMR/MSS/MSI-L. Abbreviations: dMMR, mismatch repair deficient; MSI-H, microsatellite instability-high; pMMR, mismatch repair-proficient; MSS, microsatellite stable; MSI-L, microsatellite instability-low; HR, hazard ratio; CI, confidence interval

$\mathbf{A}$

\begin{tabular}{|c|c|c|c|}
\hline \multirow[b]{2}{*}{ Study } & \multicolumn{2}{|c|}{ No. of patients } & \multirow{2}{*}{$\begin{array}{c}\text { Disease-free survival } \\
\text { hazard ratio } \\
(95 \% \mathrm{CI})\end{array}$} \\
\hline & dMMR/MSI-H & pMMR/MSS/MSI-L & \\
\hline Choi et al. (2019) & 40 & 552 & $0.31(0.14$ to 0.69$)$ \\
\hline Kim et al. (2020) & 41 & 318 & $0.50(0.21$ to 1.17$)$ \\
\hline Ramos et al. (2020) & 31 & 147 & $0.27(0.09$ to 0.78$)$ \\
\hline Tsai et al. (2020) & 83 & 846 & $0.46(0.31$ to 0.68$)$ \\
\hline Overall (Heterogenei & $0 \%)$ & & $0.42(0.31$ to 0.57$)$ \\
\hline
\end{tabular}

B

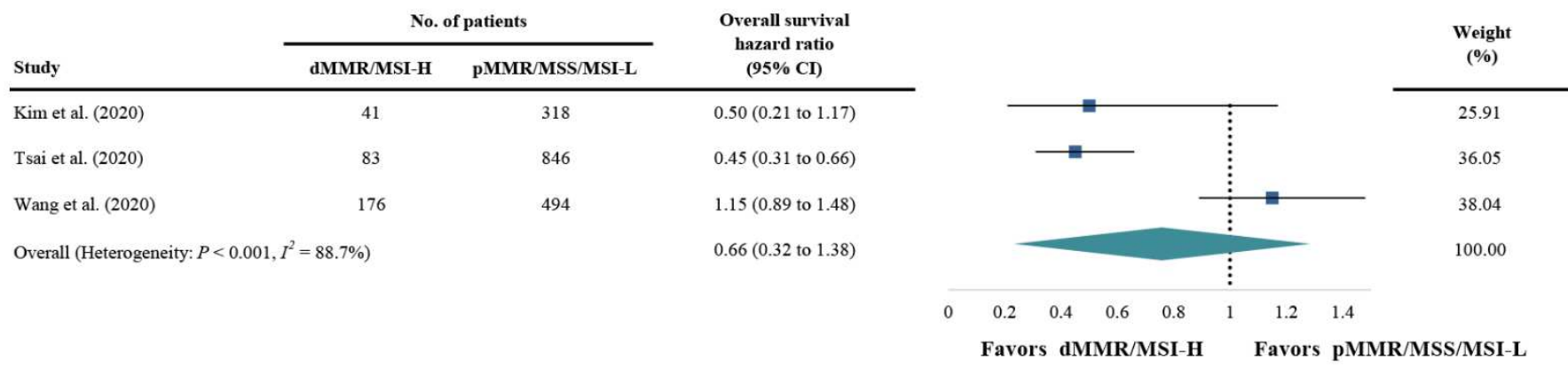

Figure 3. Meta-analysis of disease-free survival (A) and overall survival (B) for gastric cancer patients with dMMR/MSI-H versus pMMR/MSS/MSI-L. Squares represent effect size (hazard ratio [HR]) of each study, and horizontal lines represent 95\% confidence interval (CI) of HR. diamonds indicate the pooled HRs of metaanalysis. Abbreviations: dMMR, mismatch repair deficient; MSI-H, microsatellite instability-high; pMMR, 
mismatch repair-proficient; MSS, microsatellite stable; MSI-L, microsatellite instability-low
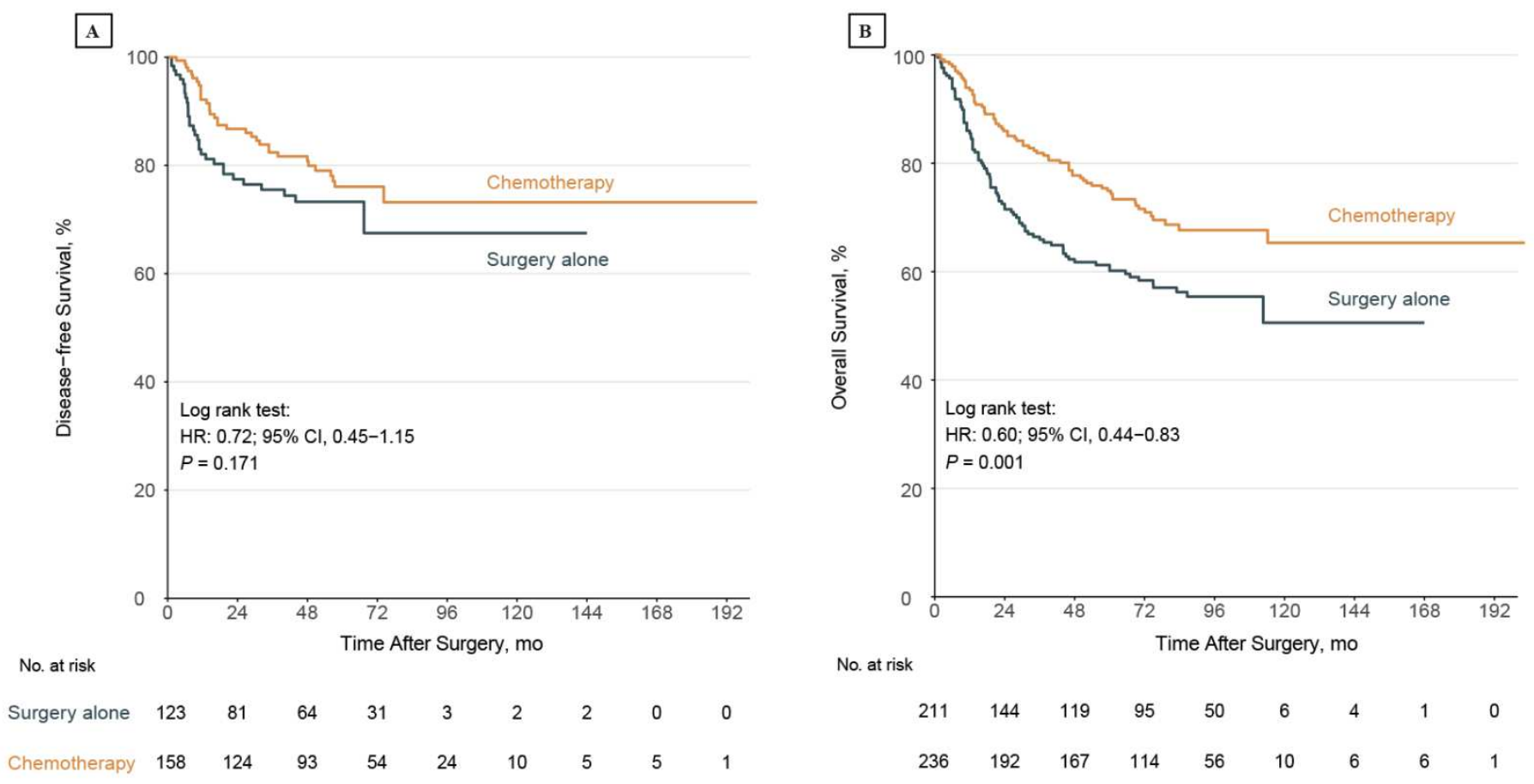

Figure 4. Individual patient data of disease-free survival (A) and overall survival (B) for gastric cancer patients with dMMR/MSI-H who received adjuvant chemotherapy versus surgery alone. Abbreviations: dMMR, mismatch repair deficient; MSI-H, microsatellite instability-high; HR, hazard ratio; CI, confidence interval

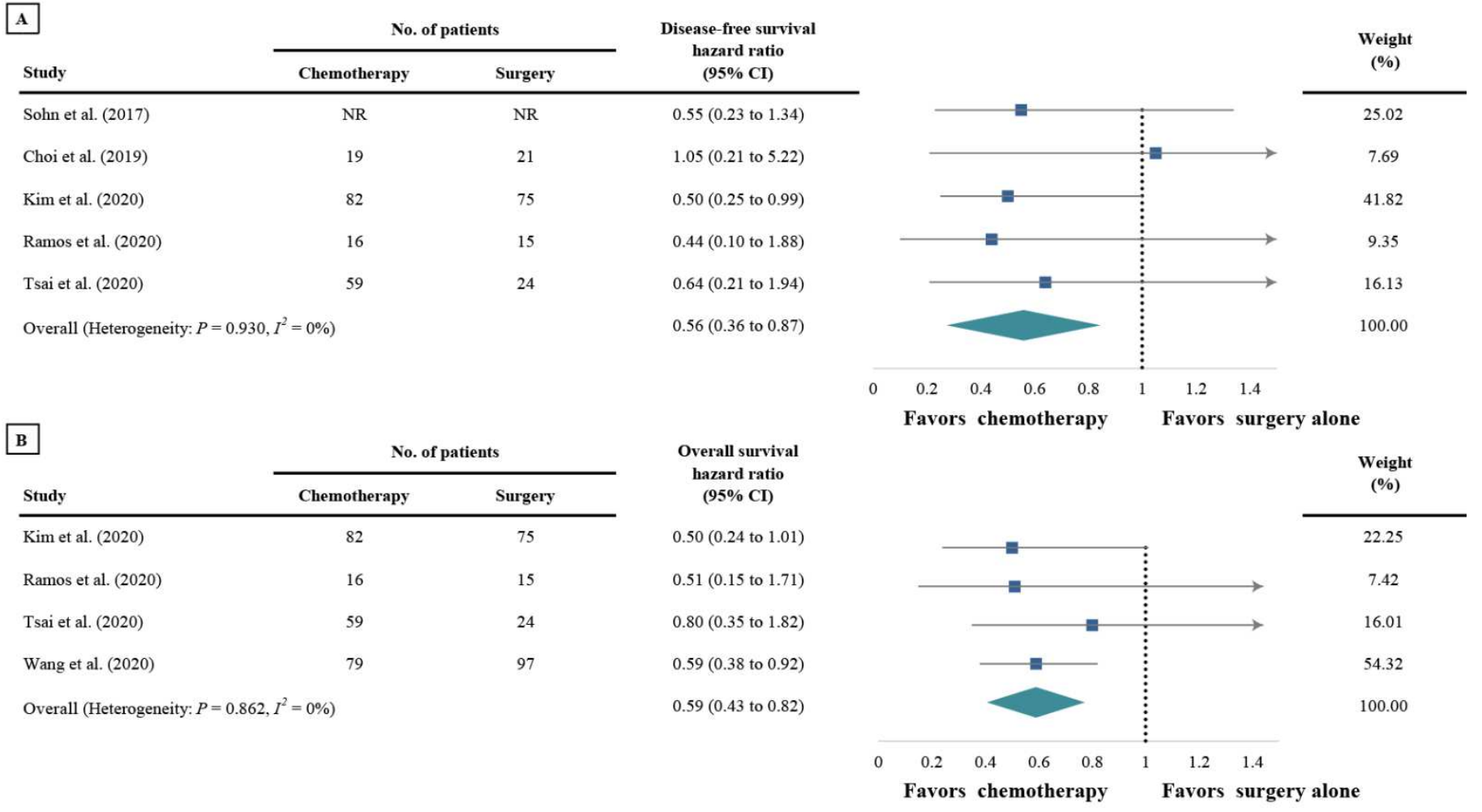

Figure 5. Meta-analysis of disease-free survival (A) and overall survival (B) for gastric cancer patients with dMMR/MSI-H who received adjuvant chemotherapy versus surgery alone. Squares represent effect size (hazard ratio [HR]) of each study, and horizontal lines represent 95\% confidence interval (CI) of HR. diamonds indicate the pooled HRs of meta-analysis. Abbreviations: dMMR, mismatch repair deficient; MSI-H, 
microsatellite instability-high

\section{Declarations}

Ethics approval and consent to participate: Not applicable

Consent for publication: Not applicable

Availability of data and materials: All data generated or analysed during this study are included in this published article.

Competing interests: No support from any organisation for the submitted work; no financial relationships with any organisations that might have an interest in the submitted work in the previous three years; no other relationships or activities that could appear to have influenced the submitted work.

Funding: None.

Author Contributions: All authors contributed to the study concept and design; R.C.N and S.Q.Y extracted the data and performed the quality assessment. R.C.N, Y.W, G.M.C, J.Z. and Y.B.C participated in analysis of the data. R.C.N, S.C., G.M.C, and J.Z. contributed to drafting of the manuscript. J.W.K., M.Y.C and Y.F.L revised the manuscript. All authors approved the final version of the manuscript.

Acknowledgements: None.

Ethical approval: Not required.

\section{REFERENCES}

1. Feng RM, Zong YN, Cao SM, Xu RH. Current cancer situation in China: good or bad news from the 2018 Global Cancer Statistics? Cancer Commun (Lond) 2019; 39: 22.

2. Bray F, Ferlay J, Soerjomataram I et al. Global cancer statistics 2018: GLOBOCAN estimates of incidence and mortality worldwide for 36 cancers in 185 countries. CA Cancer J Clin 2018.

3. Sakuramoto S, Sasako M, Yamaguchi T et al. Adjuvant chemotherapy for gastric cancer with S-1, an oral fluoropyrimidine. $\mathrm{N}$ Engl J Med 2007; 357: 1810-1820.

4. Sasako M, Sakuramoto S, Katai $\mathrm{H}$ et al. Five-year outcomes of a randomized phase III trial comparing adjuvant chemotherapy with S-1 versus surgery alone in stage II or III gastric cancer. J Clin Oncol 2011; 29: 4387-4393.

5. Bang Y-J, Kim Y-W, Yang H-K et al. Adjuvant capecitabine and oxaliplatin for gastric cancer after D2 gastrectomy (CLASSIC): a phase 3 open-label, randomised controlled trial. The Lancet 2012; 379: 315-321.

6. Noh SH, Park SR, Yang H-K et al. Adjuvant capecitabine plus oxaliplatin for gastric cancer after D2 gastrectomy (CLASSIC): 5- 
year follow-up of an open-label, randomised phase 3 trial. The Lancet Oncology 2014; 15: 1389-1396.

7. Wang FH, Shen L, Li J et al. The Chinese Society of Clinical Oncology (CSCO): clinical guidelines for the diagnosis and treatment of gastric cancer. Cancer Commun (Lond) 2019; 39: 10.

8. Yoshida K, Kodera Y, Kochi M et al. Addition of Docetaxel to Oral Fluoropyrimidine Improves Efficacy in Patients With Stage III Gastric Cancer: Interim Analysis of JACCRO GC-07, a Randomized Controlled Trial. J Clin Oncol 2019; 37: 1296-1304.

9. Cheong JH, Yang HK, Kim $\mathrm{H}$ et al. Predictive test for chemotherapy response in resectable gastric cancer: a multi-cohort, retrospective analysis. Lancet Oncol 2018; 19: 629-638.

10. Wang JB, Li P, Liu XL et al. An immune checkpoint score system for prognostic evaluation and adjuvant chemotherapy selection in gastric cancer. Nat Commun 2020; 11: 6352.

11. Karran P. Microsatellite instability and DNA mismatch repair in human cancer. Semin Cancer Biol 1996; 7: 15-24.

12. Hause RJ, Pritchard CC, Shendure J, Salipante SJ. Classification and characterization of microsatellite instability across 18 cancer types. Nature Medicine 2016; 22: 1342-1350.

13. Ribic CM, Sargent DJ, Moore MJ et al. Tumor microsatellite-instability status as a predictor of benefit from fluorouracil-based adjuvant chemotherapy for colon cancer. N Engl J Med 2003; 349: 247-257.

14. Sargent DJ, Marsoni S, Monges $G$ et al. Defective mismatch repair as a predictive marker for lack of efficacy of fluorouracilbased adjuvant therapy in colon cancer. J Clin Oncol 2010; 28: 3219-3226.

15. Marabelle A, Le DT, Ascierto PA et al. Efficacy of Pembrolizumab in Patients With Noncolorectal High Microsatellite Instability/Mismatch Repair-Deficient Cancer: Results From the Phase II KEYNOTE-158 Study. J Clin Oncol 2020; 38: 1-10.

16. Choi YY, Kim H, Shin SJ et al. Microsatellite Instability and Programmed Cell Death-Ligand 1 Expression in Stage II/III Gastric Cancer: Post Hoc Analysis of the CLASSIC Randomized Controlled study. Ann Surg 2019; 270: 309-316.

17. Pietrantonio F, Miceli R, Raimondi A et al. Individual Patient Data Meta-Analysis of the Value of Microsatellite Instability As a Biomarker in Gastric Cancer. J Clin Oncol 2019; 37: 3392-3400.

18. Kim JW, Cho SY, Chae J et al. Adjuvant Chemotherapy in Microsatellite Instability-High Gastric Cancer. Cancer Res Treat 2020; 52: 1178-1187.

19. Liberati A, Altman DG, Tetzlaff J et al. The PRISMA statement for reporting systematic reviews and meta-analyses of studies that evaluate healthcare interventions: explanation and elaboration. BMJ 2009; 339: b2700.

20. Higgins J, S G. Cochrane handbook for systematic reviews of interventions. New York, NY: Cochrane Collaboration, John Wiley and Sons. 2008.

21. GA W, B S, DO C. The Newcastle-Ottawa Scale (NOS) for assessing the quality of nonrandomised studies in meta-analyses. 2011.

22. Corsello SM, Nagari RT, Spangler RD et al. Discovering the anti-cancer potential of non-oncology drugs by systematic viability profiling. Nat Cancer 2020; 1: 235-248.

23. Sohn BH, Hwang JE, Jang HJ et al. Clinical Significance of Four Molecular Subtypes of Gastric Cancer Identified by The Cancer Genome Atlas Project. Clin Cancer Res 2017.

24. Ramos M, Pereira MA, Amorim LC et al. Gastric cancer molecular classification and adjuvant therapy: Is there a different benefit according to the subtype? J Surg Oncol 2020; 121: 804-813.

25. Tsai CY, Lin TA, Huang SC et al. Is Adjuvant Chemotherapy Necessary for Patients with Deficient Mismatch Repair Gastric Cancer?-Autophagy Inhibition Matches the Mismatched. Oncologist 2020; 25: e1021-e1030.

26. Cancer Genome Atlas Research N. Comprehensive molecular characterization of gastric adenocarcinoma. Nature 2014; 513: 202-209.

27. Polom K, Marano L, Marrelli $\mathrm{D}$ et al. Meta-analysis of microsatellite instability in relation to clinicopathological characteristics and overall survival in gastric cancer. Br J Surg 2018; 105: 159-167.

28. Zhu L, Li Z, Wang Y et al. Microsatellite instability and survival in gastric cancer: A systematic review and meta-analysis. Mol Clin Oncol 2015; 3: 699-705.

29. Le DT, Uram JN, Wang H et al. PD-1 Blockade in Tumors with Mismatch-Repair Deficiency. N Engl J Med 2015; 372: 2509-2520.

30. Le DT, Durham JN, Smith KN et al. Mismatch repair deficiency predicts response of solid tumors to PD-1 blockade. Science 2017; 357: 409-413. 
31. Andre T, Shiu KK, Kim TW et al. Pembrolizumab in Microsatellite-Instability-High Advanced Colorectal Cancer. N Engl J Med 2020; 383: 2207-2218.

32. Fuchs CS, Doi T, Jang RW et al. Safety and Efficacy of Pembrolizumab Monotherapy in Patients With Previously Treated Advanced Gastric and Gastroesophageal Junction Cancer. JAMA Oncology 2018; 4.

33. Kim ST, Cristescu R, Bass AJ et al. Comprehensive molecular characterization of clinical responses to PD-1 inhibition in metastatic gastric cancer. Nat Med 2018; 24: 1449-1458. 


\section{Figures}

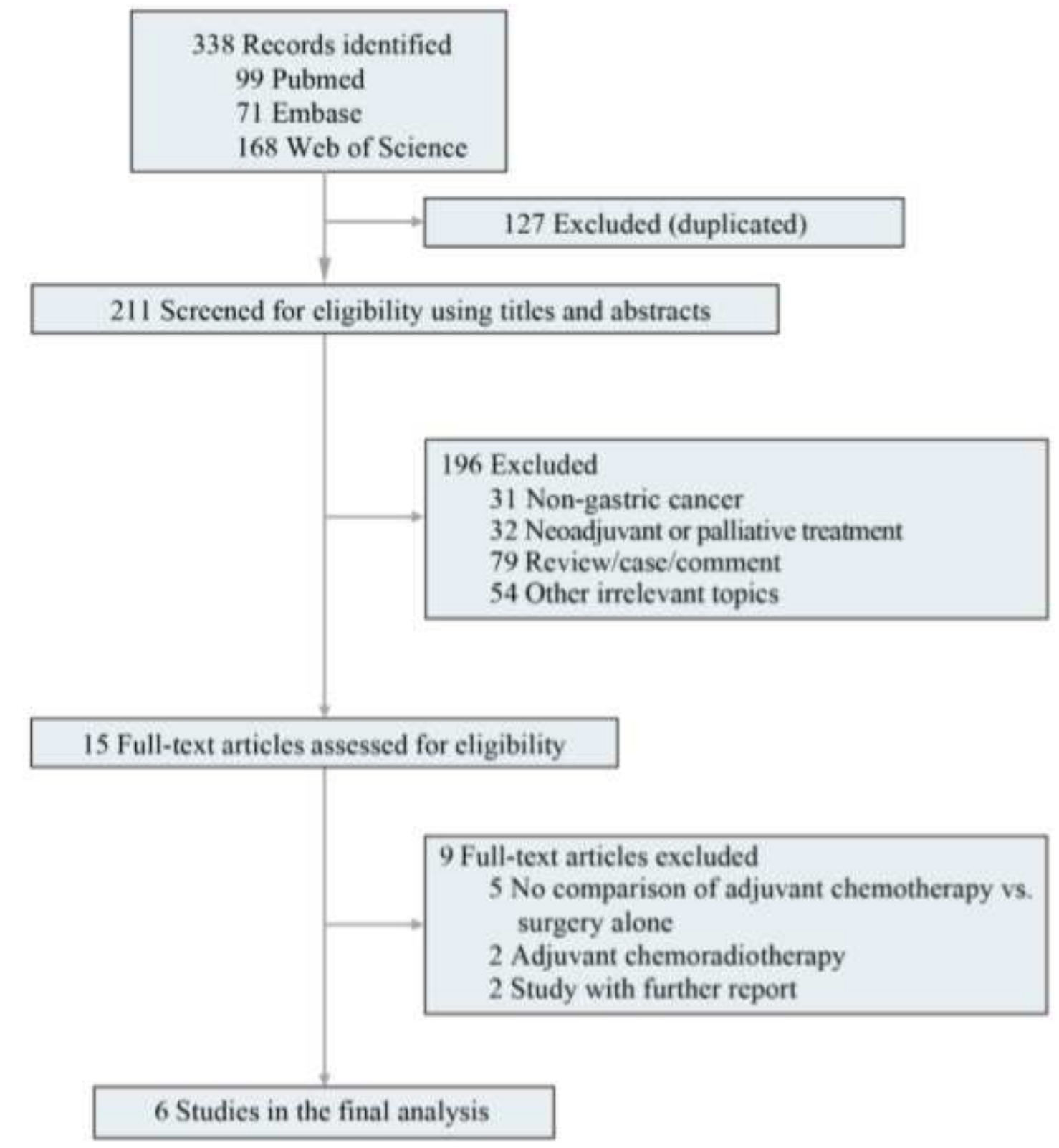

\section{Figure 1}

Study flow diagram. Six studies were identified for quantitative analysis. 
A

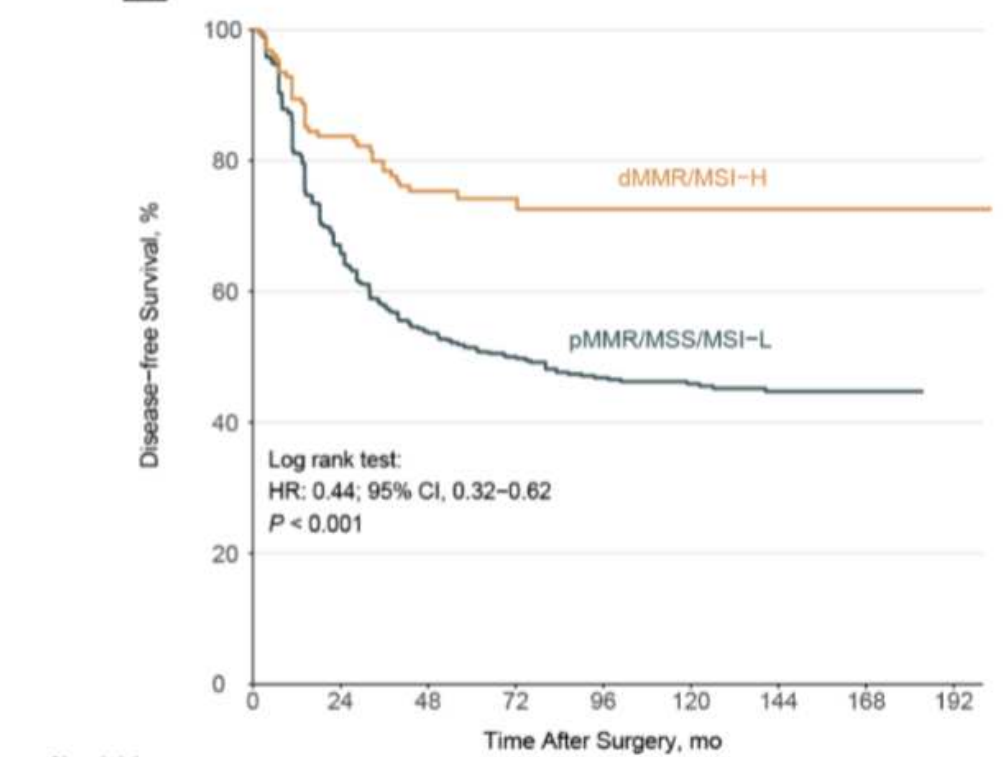

No. at risk
B

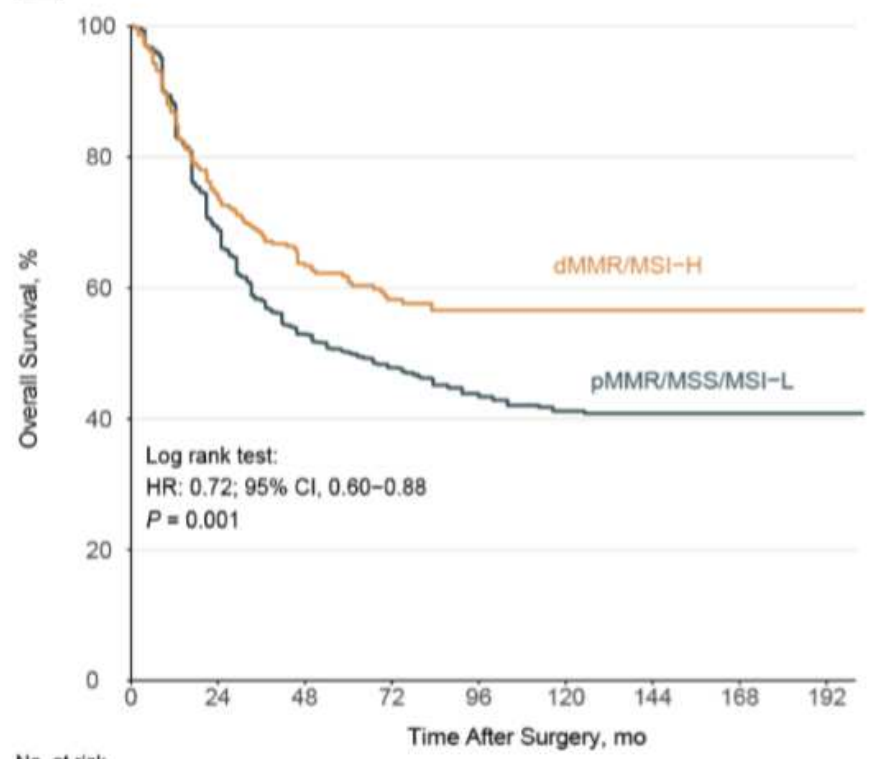

No. at risk

$\begin{array}{rrrrrrrrrr}\text { PMMR/MSS/MSI-L } & 1597 & 964 & 726 & 378 & 163 & 134 & 88 & 79 & 0 \\ & & & & & & & & & \end{array}$

\section{Figure 2}

Individual patient data of disease-free survival (A) and overall survival (B) for gastric cancer patients with dMMR/MSI-H versus pMMR/MSS/MSI-L. Abbreviations: dMMR, mismatch repair deficient; MSI-H, microsatellite instability-high; pMMR, mismatch repair-proficient; MSS, microsatellite stable; MSI-L, microsatellite instability-low; $\mathrm{HR}$, hazard ratio; $\mathrm{Cl}$, confidence interval

A

\begin{tabular}{|c|c|c|c|}
\hline \multirow[b]{2}{*}{ Study } & \multicolumn{2}{|c|}{ No. of patients } & \multirow{2}{*}{$\begin{array}{c}\text { Disease-free survival } \\
\text { hazard ratio } \\
(95 \% \text { CD) }\end{array}$} \\
\hline & dMMR/MSI-H & PMMR/MSS/MSI-L & \\
\hline Choi et al. (2019) & 40 & 552 & $0.31(0.14$ to 0.69$)$ \\
\hline Kim et al. (2020) & 41 & 318 & $0.50(0.21$ to 1.17$)$ \\
\hline Ramos et al. (2020) & 31 & 147 & $0.27(0.09$ to 0.78$)$ \\
\hline Tsai et al. (2020) & 83 & 846 & $0.46(0.31$ to 0.68$)$ \\
\hline Overall (Heterogenei & $=0 \%$ ) & & $0.42(0.31$ to 0.57$)$ \\
\hline
\end{tabular}

B

\begin{tabular}{|c|c|c|c|}
\hline \multirow[b]{2}{*}{ Study } & \multicolumn{2}{|c|}{ No. of patients } & \multirow{2}{*}{$\begin{array}{c}\text { Overall survival } \\
\text { hazard ratio } \\
\text { (95\% CD) }\end{array}$} \\
\hline & dMMR/MSI-H & pMMR/MSS/MSI-L & \\
\hline Kim et al. (2020) & 41 & 318 & $0.50(0.21$ to 1.17$)$ \\
\hline Tsai et al. (2020) & 83 & 846 & $0.45(0.31$ to 0.66$)$ \\
\hline Wang et al. (2020) & 176 & 494 & $1.15(0.89$ to 1.48$)$ \\
\hline \multicolumn{3}{|c|}{ Overall (Heterogeneity: $P<0.001, I^{2}=88.7 \%$ ) } & $0.66(0.32$ to 1.38$)$ \\
\hline
\end{tabular}

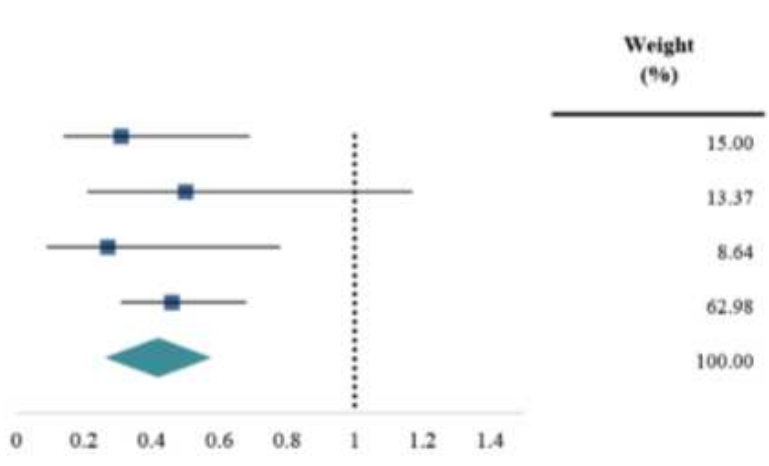

Favers dMMR/MSI-H Favors pMMR/MSS/MSI-L

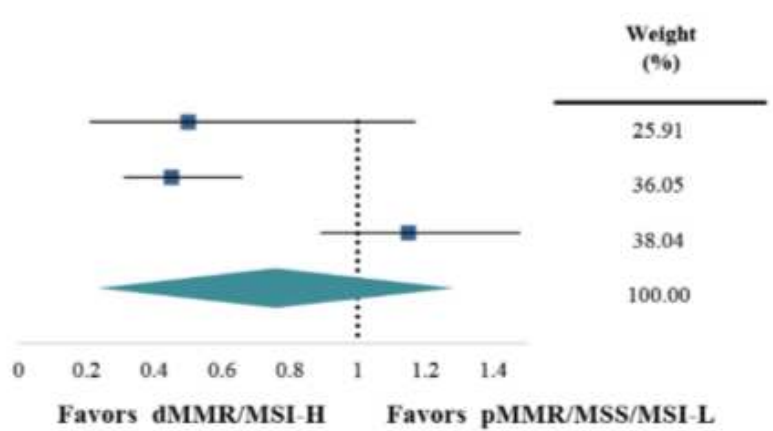




\section{Figure 3}

Meta-analysis of disease-free survival (A) and overall survival (B) for gastric cancer patients with dMMR/MSI-H versus pMMR/MSS/MSI-L. Squares represent effect size (hazard ratio [HR]) of each study, and horizontal lines represent $95 \%$ confidence interval $(\mathrm{Cl})$ of HR. diamonds indicate the pooled HRs of metaanalysis. Abbreviations: dMMR, mismatch repair deficient; $\mathrm{MSI}-\mathrm{H}$, microsatellite instability-high; pMMR, mismatch repair-proficient; MSS, microsatellite stable; MSI-L, microsatellite instability-low

$\Delta$

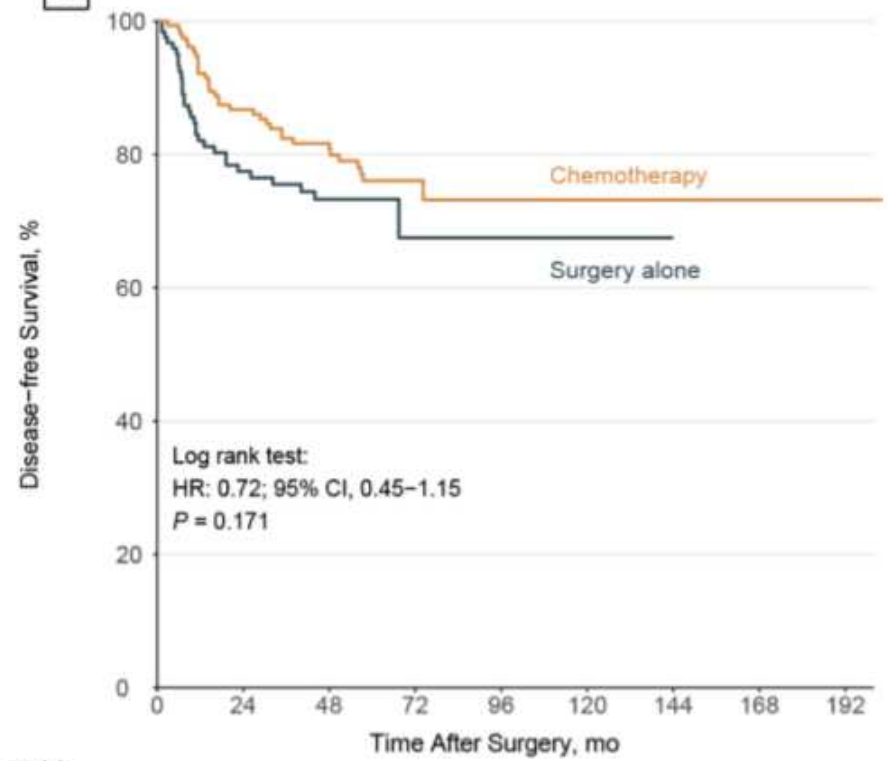

No, at risk

Surgery alone

Chemotherapy

$\begin{array}{ccccccccc}123 & 81 & 64 & 31 & 3 & 2 & 2 & 0 & 0 \\ 158 & 124 & 93 & 54 & 24 & 10 & 5 & 5 & 1\end{array}$

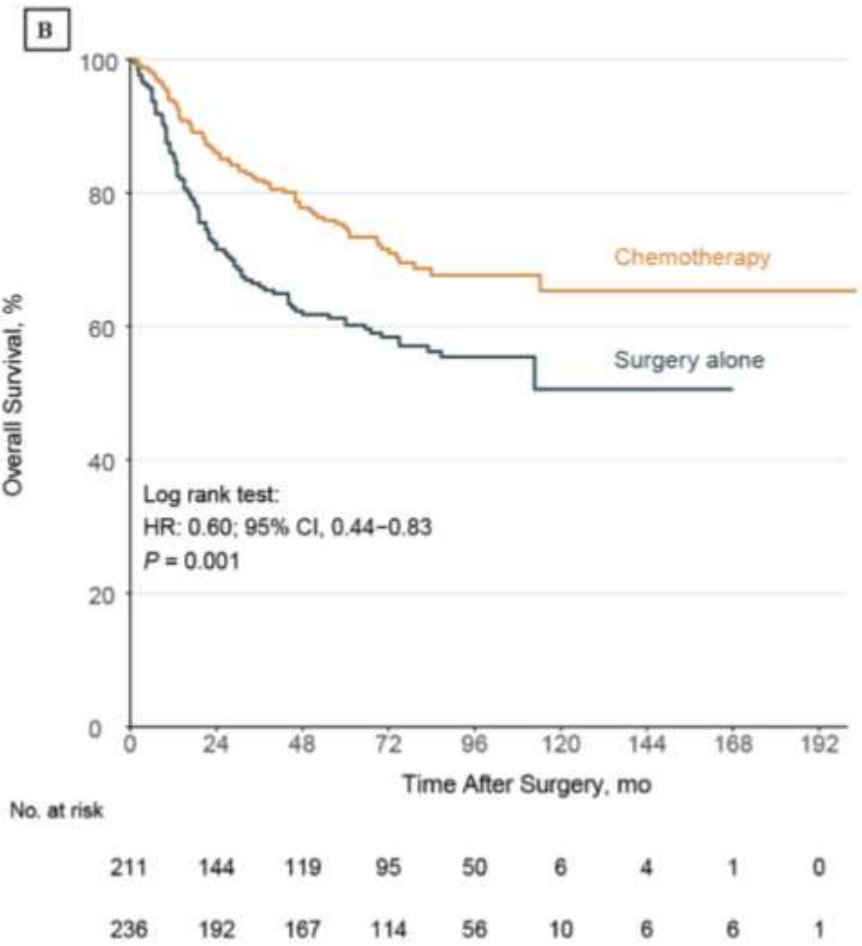

\section{Figure 4}

Individual patient data of disease-free survival (A) and overall survival (B) for gastric cancer patients with $\mathrm{dMMR/MSI-H}$ who received adjuvant chemotherapy versus surgery alone. Abbreviations: dMMR, mismatch repair deficient; $\mathrm{MSI}-\mathrm{H}$, microsatellite instability-high; $\mathrm{HR}$, hazard ratio; $\mathrm{Cl}$, confidence interval 
A

\begin{tabular}{|c|c|c|c|}
\hline \multirow[b]{2}{*}{ Study } & \multicolumn{2}{|c|}{ No. of patients } & \multirow{2}{*}{$\begin{array}{l}\text { Disease-free survival } \\
\text { hazard ratio } \\
\text { (95\% CI) }\end{array}$} \\
\hline & Chemotherapy & Surgery & \\
\hline Sohn et al. (2017) & NR & NR & $0.55(0.23$ to 1.34$)$ \\
\hline Choi et al (2019) & 19 & 21 & $1.05(0.21$ to 5.22$)$ \\
\hline Kim et al. $(2020)$ & 82 & 75 & $0.50(0.25$ to 0.99$)$ \\
\hline Ramos et al. (2020) & 16 & 15 & $0.44(0.10$ to 1.88$)$ \\
\hline Tsai et al. (2020) & 59 & 24 & $0.64(0.21$ to 1.94$)$ \\
\hline \multicolumn{3}{|c|}{ Overall (Heterogeneity: $P=0.930, I^{2}=0 \%$ ) } & $0.56(0.36$ to 0.87$)$ \\
\hline
\end{tabular}

B

\begin{tabular}{lcc} 
& \multicolumn{2}{c}{ No. of patients } \\
\cline { 2 - 3 } Study & Chemotherapy & Surgery \\
\hline Kim et al. (2020) & 82 & 75 \\
Ramos et al. (2020) & 16 & 15 \\
Tsai et al. (2020) & 59 & 24 \\
Wang et al. (2020) & 79 & 97 \\
Overall (Heterogeneity: $P=0.862, P^{2}=0 \%$ ) &
\end{tabular}

$$
\begin{gathered}
\begin{array}{c}
\text { Overall survival } \\
\text { hazard ratio } \\
\text { (95\% CI) }
\end{array} \\
0.50(0.24 \text { to } 1.01) \\
0.51(0.15 \text { to } 1.71) \\
0.80(0.35 \text { to } 1.82) \\
0.59 \text { (0.38 to } 0.92) \\
0.59 \text { (0.43 to } 0.82)
\end{gathered}
$$

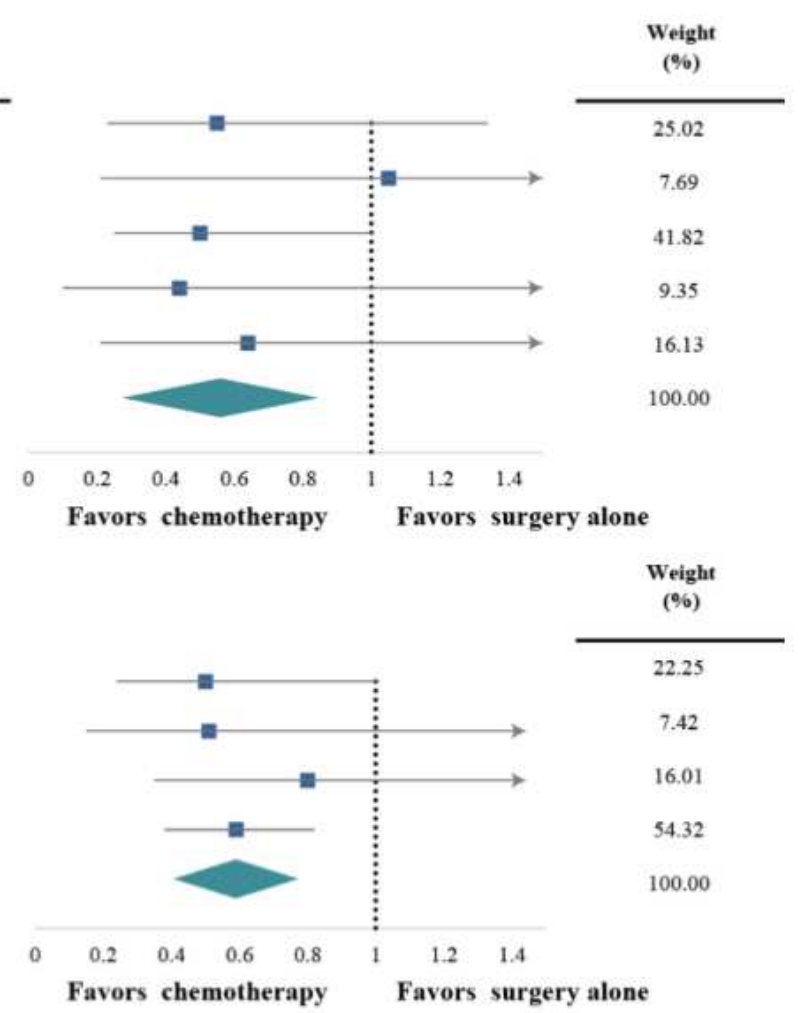

Figure 5

Meta-analysis of disease-free survival (A) and overall survival (B) for gastric cancer patients with dMMR/MSI-H who received adjuvant chemotherapy versus surgery alone. Squares represent effect size (hazard ratio [HR]) of each study, and horizontal lines represent 95\% confidence interval (Cl) of $\mathrm{HR}$. diamonds indicate the pooled HRs of meta-analysis. Abbreviations: dMMR, mismatch repair deficient; MSI-H,

\section{Supplementary Files}

This is a list of supplementary files associated with this preprint. Click to download.

- PRISMAchecklist.doc

- Supplementarymaterials.docx 